\title{
Perfil de resistência aos antimicrobianos e prevalência da produção de beta-lactamases de espectro estendido em cepas de Escherichia coli em um hospital terciário do Ceará, Brasil (2010-2013)
}

\section{Resistance profile antimicrobial and prevalence of production of beta- lactamases of extended spectrum strains of Escherichia coli in a tertiary hospital in Ceará, Brazil (2010 - 2013)}

Kathiane Lustosa Augusto ${ }^{1}$. Andrea Goiana Martins ${ }^{2}$. Leonardo Robson Pinheiro Sobreira Bezerra ${ }^{3}$. Jose Ananias Vasconcelos Neto ${ }^{4}$. Camila Teixeira Moreira Vasconcelos 5 . Sara Arcanjo Lino Karbage ${ }^{6}$. Thales Wellington Menezes Ferreira ${ }^{7}$. Nathália Cabó Façanha ${ }^{8}$. Erika Perdigão Ogawa ${ }^{9}$. Andreisa Paiva Monteiro Bilhar $^{10}$. Evelyne Santana Girão ${ }^{11}$.

1 Mestre em Ciências Clínico-cirúrgicas da Universidade Federal do Ceará (UFC), Fortaleza, Ceará, Brasil. 2 Estudante de Medicina, Universidade Estadual do Ceará (UECE), Fortaleza, Ceará, Brasil. 3 Professor do Magistério Superior, Adjunto-A da Universidade Federal do Ceará (UFC), Fortaleza, Ceará, Brasil. 4 Mestre em Tocoginecologia pela Universidade Federal do Ceará (UFC), Fortaleza, Ceará, Brasil. 5 Professora Adjunto I do Departamento de Enfermagem da Universidade Federal do Ceará (UFC), Fortaleza, Ceará, Brasil. 6 Mestre em Saúde Pública pela Universidade de Fortaleza (UNIFOR), Fortaleza, Ceará, Brasil. 7 Estudante de Medicina da Universidade Estadual do Ceará (UECE), Fortaleza, Ceará, Brasil. 8 Médica pela Universidade Estadual do Ceará (UECE), Fortaleza, Ceará, Brasil. 9 Médica pela Universidade Federal do Ceará (UFC), Fortaleza, Ceará, Brasil. 10 Ginecologista especialista em Uroginecologia pela Universidade Federal de São Paulo (UNIFESP). 11 Mestre em Ciências Médicas pelo programa de Doenças Infecciosas e Parasitárias da Faculdade de Medicina da Universidade de São Paulo (FMUSP).

\section{RESUMO}

OBJETIVOS: A resistência bacteriana aos antimicrobianos comumente utilizados na prática médica para tratamento das infecções do trato urinário (ITU) vem crescendo nos últimos anos, acarretando maiores gastos com a saúde da mulher. Os objetivos deste estudo são traçar o perfil epidemiológico dos uropatógenos e o perfil de susceptibilidade antimicrobiana desses microorganismos nas urinoculturas positivas de mulheres, acima de 18 anos, atendidas ambulatorialmente em um hospital terciário de Fortaleza entre os anos de 2010 a 2013, além de prover um perfil da produção da enzima beta-lactamase de espectro estendido (ESBL) nas cepas de E. coli isoladas nos anos de 2010 a 2012. METODOLOGIA: Realizado estudo retrospectivo transversal no Hospital Geral de Fortaleza (HGF), coletando-se um total de 2.852 urinoculturas e obtendo-se dados acerca da prevalência de uropatógenos e o perfil de susceptibilidade antimicrobiana destes microorganismos. A identificação das espécies bacterianas e testes de antibiograma foram realizados utilizando-se a metodologia automatizada Vitek 2 ( bioMérieux). A produção de betalactamases de espectro estendido também foi detectada pelo Vitek 2, seguindo os critérios do CLSI (Clinical Laboratory Standards Institute). RESULTADOS: Do total 2.852 urinoculturas positivas, 1.193 (41,8\%) eram de mulheres com idade superior a 18 anos de demanda ambulatorial. Dessas amostras, Escherichia coli foi o agente mais prevalente (59,8\%). Observa-se incidência crescente isolamento de E. coli durante o período estudado $(\mathrm{p}<0.001)$. As cepas de $E$. coli encontradas apresentaram taxas elevadas de resistência para ampicilina (de 52 a 67\%), sulfametoxazol-trimetropim ( 43 a50\%), ciprofloxacino ( 26 a 35\%) e cefalotina( 22 a $30 \%$ ) no período estudado. Do total de 713 cepas de E. coli isoladas, 71 (9,9\%) foram produtoras ESBL. Observou-se importante tendência de crescimento no período avaliado, variando de 10,7\% em 2010 a 18,6\% em 2012. CONCLUSÕES: E. coli foi o principal agente de infecção urinária em mulheres adultas na comunidade, com elevadas taxas de resistência aos antimicrobianos mais utilizados. As taxas crescentes de produção de ESBL por esse patógeno são alarmantes, dificultando o tratamento dessas infecções.

Palavras-chave: Infecções urinárias. Saúde da mulher. Coleta de urina. Testes de sensibilidade microbiana. Farmacorresistência bacteriana. 


\section{ABSTRACT}

OBJECTIVES: bacterial resistance to antimicrobials commonly used in medical practice for treatment of urinary tract infections (UTI) has been growing in recent years, leading to higher spending on women's health. The objectives of this study are to trace the epidemiological profile of uropathogens and profile of antimicrobial susceptibility of these microorganisms in positive urine cultures of women above 18 years, seen as outpatients at a tertiary hospital in Fortaleza between the years 2010-2013, and provide a profile production of beta-lactamase enzyme extended spectrum (ESBL) in E. coli strains isolated in the years 2010 to 2012. METHODOLOGY: Conducted retrospective study at the General Hospital of Fortaleza (HGF), collecting a total of 2,852 urine cultures and obtaining data on the prevalence of uropathogens and profile of antimicrobial susceptibility of these microorganisms. The identification of bacterial species and antibiotic sensitivity tests were done using automated methodology Vitek 2 (bioMérieux). The production of extended spectrum beta-lactamases was also detected by Vitek 2, following the criteria of CLSI (Clinical Laboratory Standards Institute). RESULTS: Of the 2,852 positive urine cultures, 1,193 (41.8\%) were women older than 18 years of outpatient demand. These samples, Escherichia coli was the most prevalent agent (59.8\%). It is observed incidence increasing isolation of $E$. coli during the study period $(\mathrm{p}<0.001)$. The strains of $E$. coli found showed high rates of resistance to ampicillin (52-67\%), sulfamethoxazole-trimethoprim (43 a50\%), ciprofloxacin (26-35\%) and cephalothin (22-30\%) during the study period. From a total of 713 strains of $E$. coli isolates, 71 (9.9\%) were producing ESBL. It noted a significant growth trend during this period, ranging from $10.7 \%$ in 2010 to $18.6 \%$ in 2012. CONCLUSIONS: E. coli was the main urinary tract infection agent in adult women in the community with high rates of resistance to more antimicrobials used. The increasing rates of ESBL production by this pathogen are alarming, difficult to treat these infections.

Keywords: Urinary tract infections. Women's health. Urine specimen collection. Microbial sensitivity tests. Drug resistance, bacterial. 


\section{INTRODUÇÃO}

As infecções do trato urinário (ITU) estão entre as infecções bacterianas mais comuns adquiridas na comunidade, sendo bastante prevalente nas mulheres. Estima-se atualmente que $80 \%$ das uroculturas positivas pertencem a pacientes deste sexo. ${ }^{1}$

Observa-se, nos últimos anos, um aumento de uropatógenos resistentes aos antimicrobianos (ATM) utilizados rotineiramente para tratamento das ITU, acarretando elevada morbi-mortalidade além de aumento dos custos nos cuidados à saúde. $\mathrm{O}$ uso indiscriminado de ATM tem sido o principal responsável pela seleção de cepas resistentes. ${ }^{2}$

Além das mudanças no perfil de susceptibilidade antimicrobiana, é importante conhecer o perfil epidemiológico local dos agentes causadores de ITU, possibilitando a escolha do tratamento empírico adequado dessas infecções. As enzimas betalactamases de espectro ampliado (ESBL), sintetizadas por algumas bactérias, proporcionam alta resistência aos antibióticos que possuem um anel betalactâmico em sua composição química, como penicilinas e cefalosporinas, pois agem neste anel por meio de hidrólise, inativando assim o efeito da droga. ${ }^{3}$

Estabelece-se que infecções do trato urinário baixo em mulheres jovens podem ser tratadas empiricamente com ATM de baixo espectro e por curto período de tempo. Isso prova a importância do conhecimento da suscetibilidade da maioria dos uropatógenos aos ATM, guiando assim a escolha mais adequada. No Brasil há poucos dados em relação a isso, e nem há regularidade de atualização desses dados.

Vários autores alertam para elevadas taxas de E. coli resistentes a diversos antimicrobianos, inclusive a ciprofloxacina. ${ }^{1-3}$ No entanto, são escassos estudos acerca da resistência aos antimicrobianos das cepas causadores de ITU na comunidade no nosso meio. Com esse objetivo, analisamos a prevalência dos microorganismos encontrados em urinoculturas positivas de pacientes do sexo feminino no Hospital Geral de Fortaleza, no período de 2010 a 2013, provendo, ainda, dados sobre a prevalência da produção da enzima betalactamase de espectro ampliado (ESBL) nas cepas de E. coli e a susceptibilidade destas cepas para drogas potencialmente ativas.

\section{MÉTODOS}

Trata-se de estudo retrospectivo de corte transversal baseado na analise de dados obtidos do laboratório de Microbiologia do Hospital Geral de Fortaleza (HGF), referência na atenção terciária à saúde no estado de Ceará. Foram incluídos os resultados das urinoculturas positivas de pacientes do sexo feminino, acima de 18 anos, sem limite de raça, colhidas no período de janeiro de 2010 a dezembro de 2013.

O laboratório de Microbiologia do HGF normatiza, para a realização de exames de urocultura, a coleta da primeira urina da manhã ou após a retenção urinária por um período de 2 a 3 horas. As pacientes foram, ainda, orientadas a desprezar o primeiro jato de urina, colhendo em seguida o restante do volume miccional nos frascos estéreis.

Apenas as amostras que apresentaram colônias com crescimento bacteriano igual ou acima de $10^{5} \mathrm{CFU} / \mathrm{ml}$ foram consideradas no presente estudo. A identificação das espécies bacterianas e testes de antibiograma foram realizados utilizando-se a metodologia automatizada Vitek 2 (bioMérieux). A produção de betalactamases de espectro estendido também foi detectada pelo Vitek 2, seguindo os critérios do CLSI (Clinical Laboratory Standards Institute).

Os dados foram submetidos à estatística descritiva, utilizando-se do programa SPSS (Statistical Package Social Science), versão 20.0. Utilizamos o teste do qui-quadrado para tendências lineares para avaliar a distribuição de $E$. coli, o perfil de resistência antimicrobiana e as tendências na produção de ESBL (somente das amostras de 2010-2012) ao longo dos anos estudados. O nível de significância foi $\mathrm{p}<0.05$.

\section{RESULTADOS}

Um total de 2.852 urinoculturas positivas foram liberadas no laboratório de microbiologia do Hospital Geral de Fortaleza de 2010-2013. Dessas, 1.193 (41,8\%) eram de mulheres com idade superior a 18 anos de demanda ambulatorial. Escherichia coli foi a bactéria mais prevalente $(59,8 \%)$, seguida por Klebsiella pneumonia (13,2\%), Streptococcus agalactie (9,5\%), Staphylococcus sp (4,2\%), Proteus mirabilis (3,5\%) e Enterococcus sp $(3,4 \%)$ (Tabela 1).

Tabela 1. Frequência de batcérias em urinoculturas ambulatoriais de mulheres de 2010-2013.

\begin{tabular}{lll}
\hline & Frequência & $\%$ \\
\hline Escherichia coli & 713 & 59,8 \\
Klebsiella pneumonia & 157 & 13,2 \\
Streptococcus agalactie & 113 & 9,5 \\
Staphylococcus sp. & 50 & 4,2 \\
Proteus mirabilis & 42 & 3,5 \\
Enterococcus sp. & 41 & 3,4 \\
Enterobacter sp. & 22 & 1,8 \\
Pseudomonas aeruginosa & 21 & 1,8 \\
Morganela morgana & 9 & 0,8 \\
Providencia sp. & 3 & 0,3 \\
Acinetobacter sp. & 2 & 0,2 \\
Outros & 20 & 1,7 \\
Total & 1193 & 100,0 \\
\hline
\end{tabular}

Fonte: Elaborado pelos autores. 
Observou-se incidência crescente de isolamento de $E$. coli durante o período estudado ( $\mathrm{p}<0.001$ ) (Gráfico 1). As cepas de E. coli encontradas apresentaram taxas elevadas de resistência para ampicilina (de 52 a 67\%), sulfametoxazol-trimetropim (43 a 50\%), ciprofloxacino (26 a 35\%) e cefalotina (22 a $30 \%$ ) no período estudado. Do total de 713 cepas de E. coli isoladas, $71(9,9 \%)$ foram produtoras ESBL. Observou-se importante tendência de crescimento no período avaliado, variando de $10,7 \%$ em 2010 a 18,6\% em 2012. Observou-se entre os antibióticos com perfil de alta resistência ainda uma tendência para o crescimento desta para $E$. coli apenas para os antimicrobianos sulfametoxazol-trimetropima e cefalotina $(\mathrm{p}<0.001)$ (Tabela 2). Essas cepas apresentaram também aumento significativo da resistência à amoxicilina-clavulanato de potássio $(4,6 \%$ a $5,9 \%$, de 2010 a 2013$)$ e à nitrofurantoína (1,5\% a 3,6\%, de 2010 a 2013), apesar de ainda serem considerados com perfil de baixa resistência.

De um total de 713 amostras de E. coli, 71 (9,9\%) foram ESBL positivo. A produção de beta-lactamase de espectro ampliado (ESBL) nas cepas de E. coli apresentaram um importante crescimento de 10,7\% em 2010 para 18,6\% em 2012 (p $<0.001$ ) (Gráfico 2).
Gráfico 1. Incidência de E. coli em urinoculturas de paciente ambulatoriais entre os anos estudados (2010-2013).

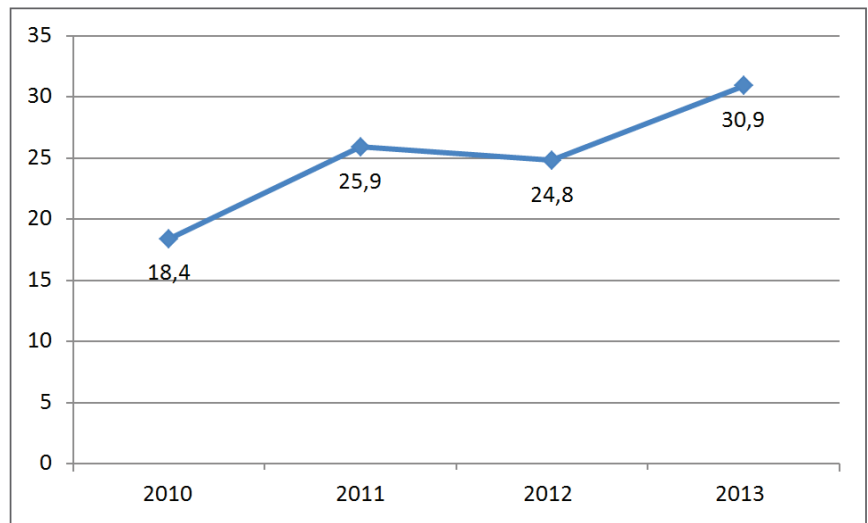

$\mathrm{x}^{2}(\mathrm{p}=0,001)$

Fonte: Elaborado pelos autores.
Gráfico 2. Tendências na produção de ESBL por E. coli ao longo dos anos estudados (2010-2013).

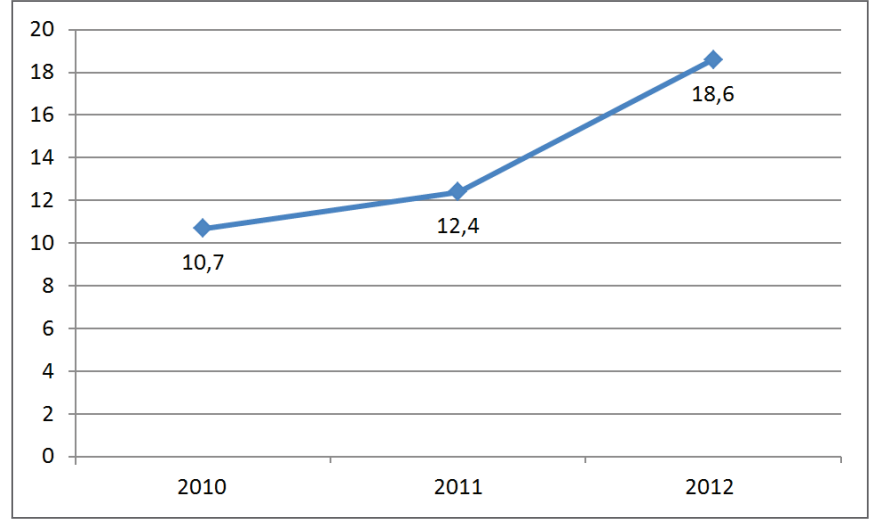

$\mathrm{x}^{2}(\mathrm{p}=0,001)$

Fonte: Elaborado pelos autores.

Tabela 2. Perfil de resistência de E. coli presente nas uroculturas positivas de pacientes ambulatoriais do sexo feminino entre os anos de 2010 a 2013.

\begin{tabular}{|c|c|c|c|c|c|c|c|c|c|}
\hline \multirow{2}{*}{ Antimicrobial } & \multicolumn{2}{|c|}{2010} & \multicolumn{2}{|c|}{2011} & \multicolumn{2}{|c|}{2012} & \multicolumn{2}{|c|}{2013} & \multirow[t]{2}{*}{$\mathrm{P}^{*}$} \\
\hline & $\mathrm{n}$ & $\% \mathrm{R}$ & $\mathrm{n}$ & $\% \mathrm{R}$ & $\mathrm{n}$ & $\% \mathrm{R}$ & $\mathrm{n}$ & $\% \mathrm{R}$ & \\
\hline Ampicilina & 83 & 63,4 & 107 & 57,8 & 93 & 52,5 & 149 & 67,7 & 0,16 \\
\hline Gentamicina & 14 & 10,7 & 18 & 9,7 & 14 & 7,9 & 23 & 10,5 & 0,91 \\
\hline Ciprofloxacino & 34 & 26 & 46 & 24,9 & 50 & 28,2 & 78 & 35,5 & 0,44 \\
\hline Sulfametoxazol-trimetoprima & 57 & 43,5 & 85 & 45,9 & 88 & 49,7 & 110 & 50 & $\mathbf{0 , 0 0}$ \\
\hline Amoxicilina - Clavulanato de potássio & 06 & 4,6 & 10 & 5,4 & 03 & 1,7 & 13 & 5,9 & 0,00 \\
\hline Cefalotina & 29 & 22,1 & 50 & 27,0 & 48 & 27,1 & 66 & 30 & $\mathbf{0 , 0 0}$ \\
\hline Ceftaxime & 13 & 9,9 & 18 & 9,7 & 17 & 9,6 & 11 & 5,0 & $\mathbf{0 , 0 0}$ \\
\hline Cefepime & 13 & 9,9 & 03 & 1,6 & 07 & 4,0 & 03 & 1,4 & $\mathbf{0 , 0 0}$ \\
\hline Ertapenem & 00 & 0,0 & 01 & 0,5 & 00 & 0,0 & 217 & 0,3 & 0,73 \\
\hline Nitrofurantoína & 02 & 1,5 & 08 & 4,3 & 06 & 3,4 & 08 & 3,6 & $\mathbf{0 , 0 0}$ \\
\hline Meropenem & 00 & 0,0 & 01 & 0,5 & 00 & 0,0 & 01 & 0,5 & 0,31 \\
\hline
\end{tabular}

Fonte: Elaborado pelos autores. 


\section{DISCUSSÃO}

Nosso estudo traça o perfil epidemiológico dos principais agentes das infecções do trato urinário de pacientes ambulatoriais do sexo feminino nos últimos anos, atendidas no Hospital Geral de Fortaleza, além do perfil de resistência antimicrobiana e a produção beta-lactamase de espectro estendido (ESBL) nas E. coli isoladas. Esses dados são de fundamental importância na prática clínica, sendo úteis na orientação dos médicos quanto ao manejo de antibióticos no tratamento empírico de ITU comunitárias naquele hospital, evitando o desenvolvimento de resistência bacteriana.

É importante destacar que nosso estudo trata-se de uma análise de resultados encontrados no laboratório de Microbiologia do HGF, encontrando limitações como a falta de conhecimento acerca do quadro clínico das pacientes que obtiveram urinoculturas positivas no período analisado, não havendo dados, portanto, para avaliar se de fato apresentavam a clínica de uma ITU, ou se apenas se tratava de contaminação de amostras ou casos de bacteriúria assintomática. Desconhecemos, ainda, se as amostras de urina positivas coletadas são realmente de ITU comunitárias não complicadas, uma vez que não obtivemos dados sobre o perfil das pacientes, se estas já haviam se submetido a uma internação hospitalar recente, se haviam feito uso recente de antimicrobianos, se utilizavam sondas vesicais que pudessem estar contaminadas ou se frequentavam redes hospitalares, como, por exemplo, pacientes em quimioterapia.

Nossos resultados encontraram uma predominância de Escherichia coli nas amostras coletadas (59,8\%), encontrando similaridade com a literatura já publicada acerca do assunto, como no estudo realizado com mulheres de demanda ambulatorial de um hospital de Jataí, em Goiás, que mostra uma frequência de $61,7 \%$ para $E$. coli e na pesquisa realizada em São Luís, no Maranhão, com análise quantitativa dos $E$. coli, dados existentes nos livros de registros das uroculturas realizadas nos meses de janeiro de 2005 a junho de 2008 no Laboratório Centrolab da rede particular de laboratórios de análises clínicas da cidade, que de um total de 565 amostras positivas para enterobactérias, 258 eram amostras colonizadas por E. coli, das quais 207 pertenciam a mulheres. ${ }^{4,5}$ Observa-se, ainda, no nosso estudo, direção de crescimento na frequência de $E$. coli durante o período estudado ( $<<0,001)$.

Os antibióticos amoxicilina-clavulanato de potássio e nitrofurantoína se mostraram os mais efetivos contra as cistites ambulatoriais causadas por $E$. coli. O pequeno número de cepas testadas para Nitrofurantoína, entretanto, dificulta a análise desse dado. Verifica-se a susceptibilidade continuada entre os anos de 2010 e 2013 das cepas de E. coli à nitrofurantoína, amoxicilina-clavulanato de potássio, amicacina, gentamicina, segunda e terceira geração de cefalosporina, ertapenem e meropenem.

Um estudo divergente aos nossos resultados foi realizado na cidade de Viçosa, em Minas Gerais, avaliando-se 113 urinoculturas colhidas em tal cidade entre junho de 2009 a maio de 2010, que apresentou uma sensibilidade a sulfametoxazoltrimetoprima de $100 \%{ }^{6}$ Em algumas outras pesquisas, também encontram-se divergências aos nossos resultados quanto à susceptibilidade de cepas $E$. coli à amoxicilinaclavulanato, como no já citado estudo realizado em São Luís, no Maranhão, que verificou uma elevada resistência de E. coli, em urinoculturas de ambos os sexos, de $75,6 \%$ a tal antibiótico. ${ }^{5,7}$

Destaca-se também nos resultados uma elevada e crescente resistência à ampicilina, fluoroquinolona, sulfametoxazoltrimetoprima e primeira geração de cefalosporina. Diversos estudos atuais realizados no Brasil evidenciam a mesma tendência de resistência antimicrobiana de cepas de E. coli.${ }^{8,9} \mathrm{Em}$ pesquisa realizada no hospital universitário da USP, de atenção secundária, em São Paulo/SP, foram colhidas urinoculturas de pacientes externos, atendidos ambulatorialmente na emergência, incluindo homens e mulheres, entre os períodos de 2005-2006 e 2010-2011, obtendo, nas amostras positivas para $E$. coli, uma susceptibilidade baixa ao sulfametoxazoltrimetoprima, apresentando $65 \%$ e $62,9 \%$ de susceptibilidade, respectivamente, nos períodos analisados. ${ }^{8}$ Ainda no mesmo estudo da USP foi encontrada uma alta resistência ( $>20 \%)$ em cepas E. coli para quinolonas (Norfloxacino e Ciprofloxacino) e cefalosporina de primeira geração (Cefazolina). ${ }^{8}$ Uma revisão bibliográfica publicada em 2014, que faz uma revisão de literatura entre os anos de 2000 a agosto de 2014 nas bases de dados Scielo e BVS a respeito dos mecanismos de resistência bacteriana na infecção urinária, orienta o uso de SMT-TMP no primeiro episódio de cistite não complicada adquirida fora do ambiente hospitalar, por ser uma droga barata e bem tolerada, porém cita outro estudo retrospectivo que foi realizado baseado no banco de dados eletrônico do hospital universitário da Fundação da Universidade Federal do Rio Grande, no qual o antibiótico que apresentou a maior prevalência da resistência bacteriana foi sulfametoxazoltrimetoprim com média $46,9 \%$, seguida por cefalotina (46,7\%), ácido nalidíxico $(27,6 \%)$ e nitrofurantoína $(22,3 \%) .{ }^{9}$

$\mathrm{O}$ alto índice de cepas resistentes ao sulfametoxazoltrimetoprim pode ter como justificativa o fato de tal antimicrobiano ser muito antigo, já tendo sido muito utilizado ao longo dos anos, possivelmente de maneira inadequada. ${ }^{10,11}$ Elevadas taxas de resistência à quinolonas e cefalosporina de primeira geração limitam o uso dessas drogas no tratamento empírico de ITU em pacientes do HGF.

A prevalência em Escherichia coli produtora de ESBL vem crescendo nos últimos anos, a despeito de uma leve queda entre os anos de 2012 e 2013, levando a uma resistência aumentada aos antimicrobianos cefalosporinas. Ainda no estudo realizado no hospital universitário da USP, em São Paulo, foi encontrada uma predominância de E. coli nas cepas produtoras de ESBL $(62,4 \%)$, seguida pelas cepas de Klebsiella spp. $(36,4 \%)$ e Proteus spp. $(1,2 \%)$, corroborando os dados encontrados no nosso estudo. ${ }^{8}$

Em estudo internacional realizado com dados obtidos de laboratórios de vinte hospitais terciários de onze países da 
América Latina no ano de 2011, 37,3\% de todas as cepas de E. coli avaliadas eram produtoras de ESBL, sendo, especificamente no Brasil, encontrada uma frequência de $18 \%$ de E. coli que sintetizam tal enzima. ${ }^{12}$ Ainda no mesmo estudo, observa-se que a cefalosporina com maior atividade contra $E$. coli é o cefepime $(72,4 \%$ de susceptibilidade, de acordo com o método CLSI). ${ }^{12}$

\section{CONCLUSÃO}

Recentemente, infecções do trato urinário (ITU) adquiridas na comunidade causadas por enterobactérias produtoras de betalactamase de espectro estendido ampliado (ESBL) têm sido descritas. Existem poucos estudos locais acerca da real prevalência da produção de ESBL por E. coli na população e dos principais uropatógenos encontrados nas infecções urinárias.

\section{REFERÊNCIAS}

1. Chambô A Filho, Camargo AS, Barbosa FA, Lopes TF, Motta YR. Estudo do perfil de resistência antimicrobiana das infecções urinárias em mulheres atendidas em hospital terciário. Rev Bras Clin Med. 2013;11(2):102-7.

2. Silva JC. Estudo retrospectivo de bactérias gram-negativas isoladas a partir de uroculturas e determinação de seu perfil de resistência. News Lab. 2014;122:82-90.

3. Junior MA, Ferreira ES, Conceição GC. Betalactamases de Espectro Ampliado (ESBL): um importante mecanismo de resistência bacteriana e sua detecção no laboratório clínico. News Lab. 2014;63:152-174.

4. Moraes D, Braoios A, Alves JL, Costa RM. Prevalência de uropatógenos e perfil de sensibilidade aos antimicrobianos em pacientes ambulatoriais de Jataí-GO. J Bras Patol Med Lab. 2014;50(3):200-4.

5. Santana TC, Maião RC, Monteiro SG, Carmos MS, Figueiredo PM. Perfil de resistência de Escherichia coli e Klebsiella spp isoladas de urocultura de comunidade do município de São Luis-Ma no período de 2005-2008. Revista de Patologia tropical. 2012;41(3):295-303.

6. Lopes PM, Queiroz TF, Rodrigues FC, Castro AS. Escherichia coli como agente etiológico de infecções do trato urinário em pacientes do município de Viçosa-MG. Rev Bras Farm. 2012;93(1):43-7.

7. Maldaner NI, Cavalli V, Rossi EM, Scapin D, Sardigla CU. Perfil
Grande parte da resistência antimicrobiana aumentada nos últimos anos é consequência do manejo clínico inadequado do uso de antibióticos na população. Uma vigilância contínua à prevalência bacteriana e ao perfil de susceptibilidade local se faz necessário visando evitar um fomento de tal resistência, diminuindo os altos custos gerados pelo uso indiscriminado das drogas e um desfecho desfavorável das ITU.

E. coli foi o principal agente de infecção urinária em mulheres adultas na comunidade, com elevadas taxas de resistência aos antimicrobianos mais utilizados. As taxas crescentes de produção de ESBL por esse patógeno são alarmantes, dificultando o tratamento dessas infecções.

antimicrobiano de cepas de Escherichia coli isolados de pessoas com suspeita de infecção do trato urinário. Rev Bras Anal Clin. 2011;43(2):145-7.

8. Miranda EJ, Oliveira GS, Roque FL, Santos SR, Olmos RD, Lotufo PA. Susceptibility to antibiotics in urinary tract infections in a secondary care setting from 2005-2006 and 2010-2011, in São Paulo, Brazil: data from 11,943 urine cultures. Rev Inst Med Trop Sao Paulo. 2014;56(4):313-24.

9. Oliveira AL, Soares MM, Santos TC, Santos A. Mecanismos de resistência bacteriana a antibióticos na infecção urinária. Revista UNINGÁ Review. 2014;20(3)65-71.

10. Bitencourt JS, Pavanelli MF. Infecção urinária em pacientes da saúde pública de Campo Mourão-PR, Brasil: prevalência bacteriana e perfil de sensibilidade. J Bras Patol Med Lab. 2014;50(5):346-51.

11. Costa LC, Belém LF, Silva, PM, Pereira HS, Silva ED Jr, Leite TR, et al. Infecções urinárias em pacientes ambulatoriais: prevalência e perfil da resistência aos antimicrobianos. Rev Bras Anal Clin. 2010;42(3):175-180.

12. Jones RN, Guzman-Blanco M, Gales AC, Gallegos B, Castro AL, Martino MD, et al. Susceptibility rates in Latin American nations: report from a regional resistance surveillance program (2011). Braz J Infect Dis. 2013;17(6):672-81.

\section{Como citar:}

Augusto KL, Martins AG, Bezerra LR, Vasconcelos JA Neto, Vasconcelos CT, Kerbage SA, et al. Perfil de resistência aos antimicrobianos e prevalência da produção de beta-lactamases de espectro estendido em cepas de Escherichia coli em um hospital terciário do Ceará, Brasil (2010 - 2013). Rev Med UFC. 2016 jan-jun;56(1):08-13. 